\title{
Editorial: Black Holes, Extended Phase Space Thermodynamics and Phase Transitions
}

\author{
Chandrasekhar Bhamidipati ${ }^{1 \dagger}$, Mohamed Chabab ${ }^{2+*}$ and Behzad Eslam Panah ${ }^{3,4,5 t}$ \\ ${ }^{1}$ School of Basic Sciences, Indian Institute of Technology Bhubaneswar, Odisha, India, ${ }^{2}$ LPHEA, Department of Physics, FSSM, \\ Cadi Ayyad University, Marrakech, Morocco, ${ }^{3}$ Department of Theoretical Physics, Faculty of Basic Sciences, University of \\ Mazandaran, Babolsar, Iran, ${ }^{4}$ ICRANet-Mazandaran, University of Mazandaran, Babolsar, Iran, ${ }^{5}$ ICRANet, Pescara, Italy
}

Keywords: black hole thermodynamics, thermal phase transitions, thermodynamic geometry, holography, ads spacetime

Editorial on the Research Topic

Black Holes, Extended Phase Space Thermodynamics and Phase Transitions

\section{OPEN ACCESS}

Edited and Reiewed by: Jan De Boer,

University of Amsterdam, Netherlands

*Correspondence:

Mohamed Chabab

mchabab@uca.ac.ma

${ }^{\dagger}$ The authors have contributed equally to this work and share first authorship

Specialty section: This article was submitted to High-Energy and Astroparticle Physics, a section of the journal Frontiers in Physics

Received: 07 May 2021 Accepted: 02 June 2021 Published: 17 June 2021

Citation: Bhamidipati C, Chabab $M$ and Eslam Panah B (2021) Editorial: Black Holes, Extended Phase Space

Thermodynamics and

Phase Transitions.

Front. Phys. 9:706197.

doi: 10.3389/fphy.2021.706197

\section{INTRODUCTION}

Over the last few decades, several remarkable connections have emerged relating gravity, thermodynamics and quantum physics. A central topic of research in gravitation which has been a rich but challenging system to make progress involves black holes as oustanding prediction of Einstein's general relativity. The recent experimental observations have raised the interest in the physics of black holes by several folds $[1,2]$. At the classical level, the black holes are defined as containing strong gravitational fields, not allowing even light to escape and emit nothing. Including quantum effects, they are not so idiosyncratic as they are governed by laws of black hole mechanics similar to the laws of thermodynamics, where: entropy, temperature and energy are related to area of the horizon, surface gravity and mass of the system, respectively [3-5]. Anti de Sitter spacetime provides a novel setting to study black holes as the thermodynamics and phase transitions can be investigated in a controlled setting due to the presence of cosmological constant. In addition, the availability of holorgraphic methods makes it very interesting to explore the physics of black holes to understand the true nature of quantum degrees of freedom of gravity [6-8]. For instance, Hawking-Page transition [9] in the AdS spacetime is related to confinement-deconfinement transitions in the boundary conformal field theory [8]. More recently, within the extended thermodynamic framework, where the cosmological constant is considered to be dynamical, providing a natural pressure in the bulk [12-18], the connection between van der Waals liquidgas transitions and phase transitions in charged black holes in AdS $[10,11]$ has been made precise, with novel concepts such as holographic heat engines [19]. These explorations have allowed us to draw novel insights into the microstructure of black holes in AdS from a macroscopic thermodynamic point of view through the methods of thermodynamic geometry [20-24].

The current special issue was born out of the need to have a collection of original articles which provide novel investigations in the aforementioned topics, which not only highlight the important recent developments, but also open up new fields for future research in extended black hole thermodynamics. It is thus interesting that new research articles have been received with contributions to topics such as, phase transitions of AdS black holes, Joule-Thomson expansion of the quasitopological black holes in the presence of some nonlinear electrodynamic models, the effects 
of dark matter on phase transition of black hole and thermodynamics of slowly accelerating black holes AdS spacetime.

In the paper Chabab $M$ and Iraoui S (2021), the continuous thermodynamic phase transitions of AdS black holes according to the generalized Ehrenfest classification have been studied. By using the Caputo fractional derivatives of thermodynamic potentials for both a charged black hole surrounded by quintessence, $5 D$ GaussBonnet and $D$ dimensional RN-AdS black holes, they find that the fractional derivatives of the Gibbs free energy is always discontinuous at the critical point for $\beta=4 / 3$ order, and diverges when $\beta>4 / 3$. These results suggest that the $4 / 3$ order phase transition is robust and holds as far one deals with a static black hole with spherical symmetry. However, this feature is not universal and fails for axisymmetric solutions, as demonstrated for Kerr black hole where the phase transition happens at $1 / 3$ order. Nevertheless, further investigations of other black holes configurations are required to consolidate these findings, and establish a more elaborated classification of thermodynamic phase transitions. Fractional phase transitions in general thermodynamic systems (including black holes) is a new and relatively unexplored topic expected to give surprising results and the preliminary results reported in the current article deserve further study.

In the paper Mirza B, Naeimipour F and Tavakoli M (2021), thermal stability and Joule-Thomson expansion of some new quasitopological black hole solutions have been investigated. At first, they have studied the higher-dimensional static quasitopological black hole solutions in the presence of BornInfeld, exponential and logarithmic models of nonlinear electrodynamic. Then, they have also obtained the fivedimensional Yang-Mills quasitopological black hole solution and compared it with the quasitopological Maxwell solution. For large values of the electric charge, and the Yang-Mills charge, they showed that the stable range of the Maxwell quasitopological black hole is larger than the Yang-Mills one. To study the JouleThomson expansion, they have considered the temperature changes in an isenthalpy process during this expansion. Their results show that the inversion curves can divide the isenthalpic ones into two parts in the inversion pressure, $P_{i}$. For $P<P_{i}$, a cooling phenomena with positive slope happens in $T-P$ diagram, while there is a heating process with negative slope for $P>P_{i}$. They also have probed the Joule-Thomson expansion of the Power Maxwell quasitopological black hole.

In the paper Singh A, Ghosh A and Bhamidipati C (2021) the effect of dark energy on the extended thermodynamic structure and interacting microstructures of black holes in AdS, through an analysis of thermodynamic geometry have been studied. Analysis of $R$ empirically reveals that dark energy parameterized by $a$, significantly alters the dominant interactions of neutral, charged and slowly rotating black hole microstructures. Above a threshold value of $\alpha, R$ is never negative at all, suggesting heuristically that the repulsive interactions due to quintessence are long ranged as opposed to the previously known short ranged repulsion in charged AdS black holes. A mean field interaction potential is proposed whose extrema effectively capture the zero crossings of Thermodynamic curvature. There are several interesting questions which need to be explored further. For instance, it needs to be understood why the thermodynamic curvature computed in different statistical ensembles does not always capture the zeros and divergences of specific heats, where as the zero crossings seem to always agree. Furthermore, although the results obtained from thermodynamic curvature are empirical in nature, is there a mechanism that can be developed or a specific example that can be studied to show that the microscopic results agree with thermodynamic expectations. There is scope for remarkable progress as the answer to some of these questions might teach us deeper aspects of quantum degrees of freedom of black holes.

Finally, in the paper Gregory R, Lim ZL and Scoins A (2021) the thermodynamics of an array of collinear black holes (which may be accelerating) have been evaluated. Also, they have proved a general First Law, including variations in the tensions of strings linking and accelerating the black holes. Then, they have analyzed the implications of the First Law in a number of instructive cases, including that of the C-metric, which relate to the previously obtained thermodynamics of slowly accelerating black holes in anti-de Sitter spacetime. The concept of thermodynamic length is found to be robust and a Christoudoulou-Ruffini formula for the C-metric is shown. There are several open questions, such as the possibility of inclusion of electric charge. Explicit solutions to Einstein-Maxwell theory describing two electrically charged black holes connected by a conical singularity, without exterior strings, are known and it would be nice to explore. Though, challenges remain as the system of multiple charged black holes destroys linearity and generalization is not straight forwarded. In addition, the issue of stability of these black holes system needs a dedicated study and is an important avenue for future research.

All the articles in this special issue and others in literature show that the field of black holes in extended thermodynamic framework is at an exciting juncture, with novel holographic connections being uncovered at a rapid pace. Apart from several research directions which have emerged as summarized above, it should be mentioned that the correct holographic interpretation of the extended thermodynamics of black holes is not available yet, and this deserves to be explored further. Another pertinent question is that although progress is being made in having pressure and volume terms in the first law of black holes in AdS space-times via different mechanisms, similar applications to asymptotically flat and de Sitter space-times need to be explored as well. All these are important questions and hope the current collection of articles is found useful in near future in this endeavor, besides serving as a reference volume in literature.

\section{AUTHOR CONTRIBUTIONS}

All authors listed have made a substantial, direct, and intellectual contribution to the work and approved it for publication.

\section{FUNDING}

CB thanks the Science and Engineering Research Board (SERB, DST), Government of India, for MATRICS (Mathematical Research Impact Centric Support) Grant No. MTR/2020/000135. 


\section{REFERENCES}

1. Abbott BP, Abbott R, Abbott TD, Acernese F, Ackley K, Adams C, et al. GW170104: Observation of a 50-Solar-Mass Binary Black Hole Coalescence at Redshift 0.2. Phys Rev Lett 118, 221101 (2017).

2. Akiyama K, Alberdi A, Alef W, Asada K, Azulay R, Baczko A-K, et al. First M87 Event Horizon Telescope Results. I. The Shadow of the Supermassive Black Hole. Astrophys J Lett 875, L1 (2019).

3. Bardeen JM, Carter B, and Hawking SW. The Four Laws of Black Hole Mechanics. Commun. Math Phys (1973) 31:161-70. doi:10.1007/bf01645742

4. Bekenstein JD. Black Holes and Entropy. Phys Rev D (1973) 7:2333-46. doi:10.1103/physrevd.7.2333

5. Hawking SW. Particle Creation by Black Holes. Commun Math Phys (1975) 43:199-220. doi:10.1007/bf02345020

6. Maldacena J. The Large $\$ \mathrm{~N} \$$ Limit of Superconformal Field Theories and Supergravity. Adv Theor Math Phys (1998) 2:231-52. doi:10.4310/ atmp.1998.v2.n2.a1

7. Gubser SS, Klebanov IR, and Polyakov AM. Gauge Theory Correlators from Non-critical String Theory. Phys Lett B (1998) 428:105-14. doi:10.1016/s03702693(98)00377-3

8. Witten E. Anti-de Sitter Space and Holography. Adv Theor Math Phys (1998) 2:253-91. doi:10.4310/ATMP.1998.v2.n2.a2

9. Hawking SW, and Page DN. Thermodynamics of Black Holes in Anti-de Sitter Space. Commun Math Phys (1983) 87:577-88. doi:10.1007/bf01208266

10. Chamblin A, Emparan R, Johnson CV, and Myers RC. Phys Rev D (1999) 60: 064018. doi:10.1103/physrevd.60.064018

11. Caldarelli MM, Cognola G, and Klemm D. Thermodynamics of KerrNewman-AdS Black Holes and Conformal Field Theories. Class Quan Grav (2000) 17:399-420. doi:10.1088/0264-9381/17/2/310

12. Kastor D, Ray S, and Traschen J. Enthalpy and the Mechanics of AdS Black Holes. Class Quan Grav (2009) 26:195011. doi:10.1088/0264-9381/26/19/195011

13. Cvetic M, Gibbons G, Kubiznak D, and Pope C. Black Hole Enthalpy and an Entropy Inequality for the Thermodynamic Volume. Phys Rev D (2011) 84: 024037. doi:10.1103/PhysRevD.84.024037

14. Dolan BP. The Cosmological Constant and Black-Hole Thermodynamic Potentials. Class Quan Grav (2011) 28:125020. doi:10.1088/0264-9381/28/12/125020
15. Kubiznak D, and Mann RB. P-V Criticality of Charged AdS Black Holes. JHEP (2012) 07:033. doi:10.1007/JHEP07(2012)033

16. Belhaj A, Chabab M, Moumni HE, and Sedra MB. On Thermodynamics of AdS Black Holes in Arbitrary Dimensions. Chin Phys Lett (2012) 29:100401. doi:10.1088/0256-307x/29/10/100401

17. Cai R-G, Cao L-M, Li L, and Yang R -Q. P-V Criticality in the Extended Phase Space of Gauss-Bonnet Black Holes in AdS Space. JHEP (2013) 09:1. doi:10.1007/JHEP09(2013)005

18. Hendi SH, Mann RB, Panahiyan S, and Eslam Panah B. Van Der Waals Like Behavior of Topological AdS Black Holes in Massive Gravity. Phys Rev D (2017) 95:(R):021501. doi:10.1103/physrevd.95.021501

19. Johnson CV. Holographic Heat Engines. Class Quan Grav (2014) 31:205002. doi:10.1088/0264-9381/31/20/205002

20. Ruppeiner G. Riemannian Geometry in Thermodynamic Fluctuation Theory. Rev Mod Phys (1995) 67:605-59. doi:10.1103/revmodphys.67.605

21. Quevedo H. Geometrothermodynamics. J Math Phys (2007) 48:013506. doi:10.1063/1.2409524

22. Hendi SH, Panahiyan S, Panah BE, and Momennia M. A New Approach toward Geometrical Concept of Black Hole Thermodynamics. Eur Phys J C (2015) 75:507. doi:10.1140/epjc/s10052-015-3701-5

23. Wei SW, Liu YX, and Mann RB. Repulsive Interactions and Universal Properties of Charged Anti-de Sitter Black Hole Microstructures. Phys Rev Lett (2019) 123(7):071103. doi:10.1103/physrevlett.123.071103

24. Ghosh A, and Bhamidipati C. Thermodynamic Geometry for Charged GaussBonnet Black Holes in AdS Spacetimes. Phys Rev D (2020) 101:4-046005. doi:10.1103/physrevd.101.046005

Conflict of Interest: The authors declare that the research was conducted in the absence of any commercial or financial relationships that could be construed as a potential conflict of interest.

Copyright $\odot 2021$ Bhamidipati, Chabab and Eslam Panah. This is an open-access article distributed under the terms of the Creative Commons Attribution License (CC BY). The use, distribution or reproduction in other forums is permitted, provided the original author(s) and the copyright owner(s) are credited and that the original publication in this journal is cited, in accordance with accepted academic practice. No use, distribution or reproduction is permitted which does not comply with these terms. 